\title{
Direct Detection Constraints on Dark Photons with the CDEX-10 Experiment at the China Jinping Underground Laboratory
}

Z. She, ${ }^{1}$ L. P. Jia, ${ }^{1}$ Q. Yue $\odot,{ }^{1, *}$ H. Ma,${ }^{1, \dagger}$ K. J. Kang, ${ }^{1}$ Y. J. Li, ${ }^{1}$ M. Agartioglu, ${ }^{2,3,8}$ H. P. An, ${ }^{4}$ J. P. Chang, ${ }^{5}$ J. H. Chen, ${ }^{2, \S}$ Y. H. Chen, ${ }^{6}$ J. P. Cheng,,${ }^{1,7}$ W. H. Dai, ${ }^{1}$ Z. Deng, ${ }^{1}$ X. P. Geng, ${ }^{1}$ H. Gong, ${ }^{1}$ P. Gu, ${ }^{8}$ Q. J. Guo, ${ }^{9}$ X. Y. Guo, ${ }^{6}$ L. He, ${ }^{5}$ S. M. He, ${ }^{6}$ H. T. He, ${ }^{8}$ J. W. Hu, ${ }^{1}$ T. C. Huang, ${ }^{10}$ H. X. Huang, ${ }^{11}$ H. B. Li, ${ }^{2,}$ H. Li, ${ }^{5}$ J. M. Li,${ }^{1}$ J. Li, ${ }^{1}$ M. X. Li,${ }^{8}$ X. Li, ${ }^{11}$ X. Q. Li ${ }^{12}$ Y. L. Li, ${ }_{1}^{1}$ B. Liao, ${ }^{7}$ F. K. Lin, ${ }^{2,}{ }^{8}$ S. T. Lin, ${ }^{8}$ S. K. Liu, ${ }^{8}$ Y. D. Liu, ${ }^{7}$ Y. Y. Liu, ${ }^{7}$ Z. Z. Liu, ${ }^{1}$ Y. C. Mao, ${ }^{9}$ Q. Y. Nie, ${ }^{1}$ J. H. Ning, ${ }^{6}$ H. Pan, ${ }^{5}$ N. C. Qi ${ }^{6}$ C. K. Qiao, ${ }^{8}$ J. Ren, ${ }^{11}$ X. C. Ruan, ${ }^{11}$ B. Sevda, ${ }^{2,3,8}$ C. S. Shang, ${ }^{6}$ V. Sharma, ${ }^{2,13,8}$ L. Singh, ${ }^{2,13,8}$ M. K. Singh, ${ }^{2,13,8}$ T. X. Sun, ${ }^{7}$ C. J. Tang, ${ }^{8}$ W. Y. Tang, ${ }^{1}$ Y. Tian, ${ }^{1}$ G. F. Wang, ${ }^{7}$ L. Wang, ${ }^{14}$ Q. Wang, ${ }^{1,4}$ Y. Wang, ${ }^{1,4}$ Y. X. Wang, ${ }^{9}$ Z. Wang, ${ }^{8}$ H. T. Wong, ${ }^{2,8}$ S. Y. Wu, ${ }^{6}$ H. Y. Xing, ${ }^{8}$ Y. Xu, ${ }^{12}$ T. Xue, ${ }^{1}$ Y. L. Yan, ${ }^{8}$ L. T. Yang, ${ }^{1,}$ N. Yi, ${ }^{5}$ C. X. Yu, ${ }^{12}$ H. J. Yu, ${ }^{5}$ J. F. Yue, ${ }^{6}$ M. Zeng, ${ }^{1}$ Z. Zeng, ${ }^{1}$ B. T. Zhang, ${ }^{1}$ L. Zhang, ${ }^{8}$ F. S. Zhang, ${ }^{7}$ Z. Y. Zhang, ${ }^{1}$

M. G. Zhao, ${ }^{12}$ J. F. Zhou, ${ }^{6}$ Z. Y. Zhou, ${ }^{11}$ and J. J. Zhu ${ }^{8}$

(CDEX Collaboration)

\author{
${ }^{1}$ Key Laboratory of Particle and Radiation Imaging (Ministry of Education) and Department of Engineering Physics, \\ Tsinghua University, Beijing 100084 \\ ${ }^{2}$ Institute of Physics, Academia Sinica, Taipei 11529 \\ ${ }^{3}$ Department of Physics, Dokuz Eylül University, İzmir 35160 \\ ${ }^{4}$ Department of Physics, Tsinghua University, Beijing 100084 \\ ${ }^{5}$ NUCTECH Company, Beijing 100084 \\ ${ }^{6}$ YaLong River Hydropower Development Company, Chengdu 610051 \\ ${ }^{7}$ College of Nuclear Science and Technology, Beijing Normal University, Beijing 100875 \\ ${ }^{8}$ College of Physics, Sichuan University, Chengdu 610064 \\ ${ }^{9}$ School of Physics, Peking University, Beijing 100871 \\ ${ }^{10}$ Sino-French Institute of Nuclear and Technology, Sun Yat-sen University, Zhuhai, 519082 \\ ${ }^{11}$ Department of Nuclear Physics, China Institute of Atomic Energy, Beijing 102413 \\ ${ }^{12}$ School of Physics, Nankai University, Tianjin 300071 \\ ${ }^{13}$ Department of Physics, Banaras Hindu University, Varanasi 221005 \\ ${ }^{14}$ Department of Physics, Beijing Normal University, Beijing 100875
}

(Received 30 October 2019; accepted 26 February 2020; published 17 March 2020)

\begin{abstract}
We report constraints on the dark photon effective kinetic mixing parameter $(\kappa)$ with data taken from two $p$-type point-contact germanium detectors of the CDEX-10 experiment at the China Jinping Underground Laboratory. The $90 \%$ confidence level upper limits on $\kappa$ of solar dark photon from $205.4 \mathrm{~kg}$-day exposure are derived, probing new parameter space with masses $\left(m_{V}\right)$ from 10 to $300 \mathrm{eV} / c^{2}$ in direct detection experiments. Considering dark photon as the cosmological dark matter, limits at $90 \%$ confidence level with $m_{V}$ from 0.1 to $4.0 \mathrm{keV} / c^{2}$ are set from $449.6 \mathrm{~kg}$-day data, with a minimum of $\kappa=1.3 \times 10^{-15}$ at $\mathrm{m}_{\mathrm{V}}=200 \mathrm{eV} / \mathrm{c}^{2}$.
\end{abstract}

DOI: 10.1103/PhysRevLett.124.111301

Introduction.-Extensive cosmological and astrophysical observations at different scales provide compelling evidence on the existence of missing matter in the Universe [1]. This motivates intense efforts to study

Published by the American Physical Society under the terms of the Creative Commons Attribution 4.0 International license. Further distribution of this work must maintain attribution to the author(s) and the published article's title, journal citation, and DOI. Funded by SCOAP ${ }^{3}$. extensions to the standard model (SM) [2]. An attractive class introduces the dark photon (denoted as $\gamma^{*}$ in this Letter) with mass $\left(m_{V}\right)$ of $\mathrm{keV} / \mathrm{c}^{2}$ scale as a potential hidden particle as a dark matter (DM) candidate $[3,4]$. The $\gamma^{*}$ can, in addition, be a new interaction mediator between DM and the SM particles [5,6]. The properties of $\gamma^{*}$, and in particular its interaction with SM particles, are parametrized by its effective kinetic mixing parameter $(\kappa)$ with the SM photons [7].

Intense gamma sources therefore provide an excellent platform to look for $\gamma^{*}$. Searches are conducted using 
high-power laser [8,9] or helioscopes [10] or via the studies of photons from stars [11] and cosmic microwave background $[5,12]$. Other studies are based on data taken with underground DM and neutrino experiments [13-20].

CDEX-10 experiment.-The second phase of the China Dark Matter Experiment (CDEX-10), with scientific goals of searching for light DM, is based on the 10-kg p-type point contact germanium (PPCGe) detector array at the China Jinping Underground Laboratory (CJPL) with a rock overburden of 2400 meters [21-25]. The detector array, consisting of three triple-element PPCGe detector strings (named C10-A, B, C, respectively), surrounded by the $20 \mathrm{~cm}$ thick high-purity oxygen-free copper as a passive shield against ambient radioactivity, is directly immersed in liquid nitrogen $\left(\mathrm{LN}_{2}\right)$ for cooling. The $\mathrm{LN}_{2}$ cryostat, with an outer diameter of $1.5 \mathrm{~m}$ and a height of $1.9 \mathrm{~m}$, together with the electronics readout and the data acquisition (DAQ) systems, is placed inside a shielding with $1 \mathrm{~m}$ thick polyethylene walls at CJPL-I [26,27]. The DAQ system consists of five readout channels with specific shaping and gain parameters for each PPCGe detector to record signal information. The configuration of the detector system was described in detail previously [26,27]. In this Letter, a raw dataset with exposure time of 363.9 day from two of the CDEX-10 detectors, named, C10-B1 and C10-C1, are used for $\gamma^{*}$ analysis.

Data taking with C10-B1 and C10-C1 was performed from February 2017 to August 2018. Independent dataquality filters were carried out for the two detectors. The resulting data with good qualities have 234.6 running days for $\mathrm{C} 10-\mathrm{B} 1$ and 276.8 running days for $\mathrm{C} 10-\mathrm{C} 1$, respectively. The dead times of the DAQ plus the veto time due to the reset signals of the preamplifiers were measured by the random trigger events to be $5.7 \%$ and $5.5 \%$ of the survival data of the two detectors, respectively. Energy calibrations were done by the zero energy (defined by the random trigger events) and the internal cosmogenic $K$-shell x-ray peaks: $8.98 \mathrm{keVee}$ of ${ }^{65} \mathrm{Zn}$ and $10.37 \mathrm{keVee}$ of ${ }^{68,71} \mathrm{Ge}$. The data preprocessing procedures for a single detector were described in previous papers [26,27], including energy calibrations, pedestal cut, physics event selection, and bulk or surface event discrimination [28]. The live times were reduced by $1.1 \%$ for $\mathrm{C} 10-\mathrm{B} 1$ and $0.6 \%$ for $\mathrm{C} 10-\mathrm{C} 1$ after pedestal cuts, which were energy independent and measured by the survival of the random trigger events. The residual cross talks between the two detectors were studied by the trigger time information. Coincident events in both detectors are extremely few in the energy region of interests and can be negligible for this study. The dead layer thicknesses of these two detectors are set to be $0.88 \pm 0.12 \mathrm{~mm}$, considering the same crystal size, crystal structure, and fabrication procedure as the CDEX-1B detector [29]. This gives rise to a fiducial mass of $939 \mathrm{~g}$ and, accordingly, valid physics data of 205.4 and $244.2 \mathrm{~kg}-$ day for $\mathrm{C} 10-\mathrm{B} 1$ and $\mathrm{C} 10-\mathrm{C} 1$, respectively.

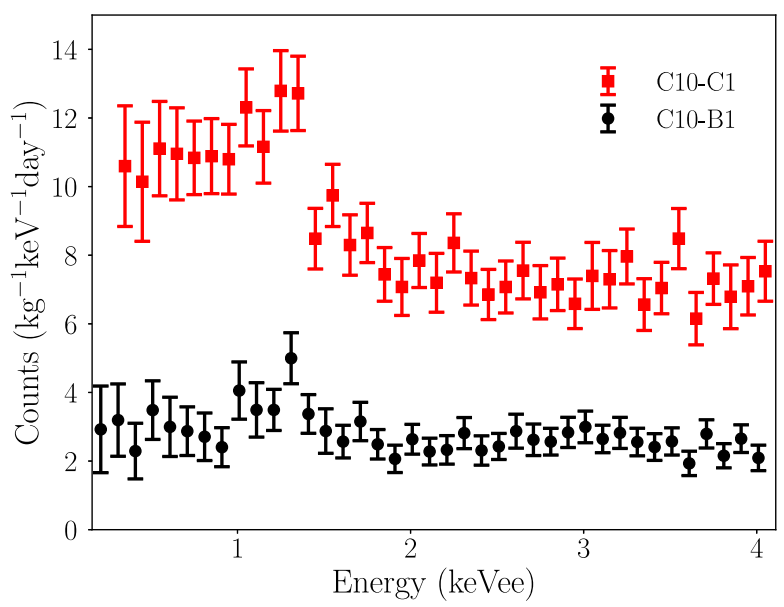

FIG. 1. The measured energy spectra of C10-B1 and C10-C1 germanium detectors at exposure of 205.4 and $244.2 \mathrm{~kg}$-day, respectively.

The energy spectra of these detectors for $\gamma^{*}$ analysis are shown in Fig. 1. The analysis thresholds are 160 eVee ("eVee" represents electron equivalent energy) for C10-B1 and 300 eVee for C10-C1. The energy peaks around $1.2 \mathrm{keVee}$ are originated from the $L$-shell $\mathrm{x}$ rays of the internal cosmogenic radionuclides. The event rates are about 2.5 and 7.6 counts kg ${ }^{-1} \mathrm{keVee}^{-1} \mathrm{day}^{-1}$ in the $2-4 \mathrm{keVee}$ energy range for these two detectors. The higher analysis threshold and event rate make the C10-C1 less sensitive to the study of solar $\gamma^{*}$, such that only $205.4 \mathrm{~kg}$-day data of C10-B1 are used in this analysis. For $\gamma^{*}$ DM analysis, however, data of these two detectors are combined to produce a larger exposure of $449.6 \mathrm{~kg}$-day.

Data analysis.-A minimal- $\chi^{2}$ analysis method, following our previous WIMPs analysis [26,30], is applied to probe $\left(m_{V}, \kappa\right)$, in which $\chi^{2}$ is defined as

$$
\chi^{2}\left(m_{V}, \kappa\right)=\sum_{j=1,2} \sum_{i=0}^{N_{j}} \frac{\left[n_{i, j}-S_{i}\left(m_{V}, \kappa\right)-B_{i, j}\right]^{2}}{\sigma_{\text {stat }, i, j}^{2}+\sigma_{\text {syst }, i, j}^{2}},
$$

where $n_{i, j}$ is the measured event rate corresponding to the $i$ th energy bin and the $j$ th detector $(j=1$ for C10-B 1 and $j=2$ for $\mathrm{C} 10-\mathrm{C} 1)$, and $S_{i}\left(m_{V}, \kappa\right)$ is the expected rate at certain $m_{V}$ and $\kappa$. The uncertainties $\sigma$ include both statistical (stat) and systematical (syst) components denoted as subscripts. $B_{i, j}$ denotes the background contribution assuming for $\mathrm{C} 10-\mathrm{B} 1$ to be a flat continuum plus several known background $L$-shell x-ray peaks, including ${ }^{68} \mathrm{Ge}$ $(1.298 \mathrm{keV}),{ }^{68} \mathrm{Ga}(1.194 \mathrm{keV}),{ }^{65} \mathrm{Zn}(1.096 \mathrm{keV}),{ }^{54} \mathrm{Mn}$ $(0.695 \mathrm{keV})$, and ${ }^{49} \mathrm{~V}(0.564 \mathrm{keV})$, for C10-B1 [26]. The intensities of these peaks from $L$-shell $\mathrm{x}$ rays are derived from those of measured $K$-shell x-ray peaks at a higher energy range of 4-12 keVee corrected by the known $\mathrm{K} / \mathrm{L}$ ratios $[26,31]$. However, the background form for C10-C1 


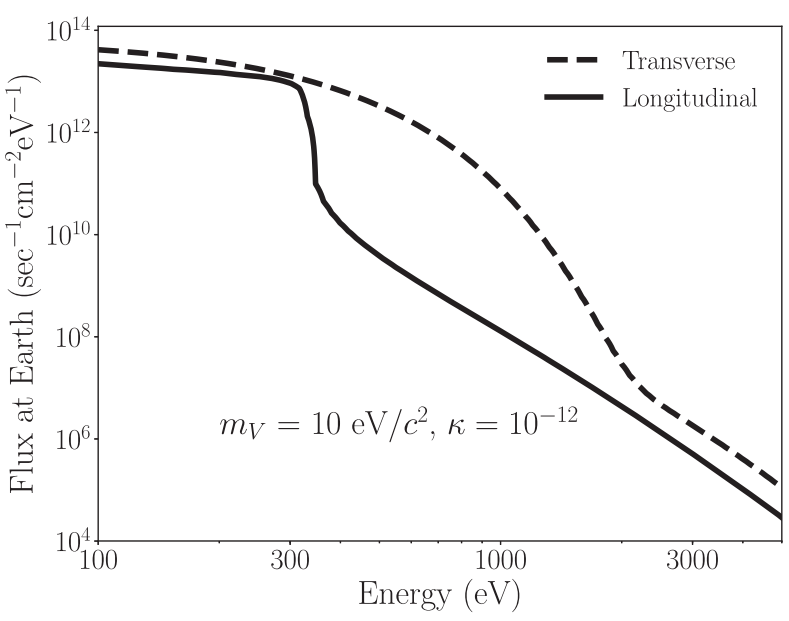

FIG. 2. The differential fluxes of solar $\gamma^{*}$ on Earth originated in the Stueckelberg case. The dashed line represents the transverse $\gamma^{*}$, while the longitudinal one is shown as a solid line.

is slightly different, so that the flat continuum profile is replaced by a linear one.

The best estimator of $\kappa$ (denoted with $\hat{\kappa}$ ) at certain $m_{V}$ is evaluated by minimizing the $\chi^{2}$ values, from which upper limits at $90 \%$ confidence level (C.L.) are derived [32]. The background contributions $B_{i, j}$ are restricted to be positive definite.

Solar dark photon.-The most significant gamma source seen on Earth is the Sun and solar $\gamma^{*}$ can be probed in underground low-background experiments and cosmological observations. The solar $\gamma^{*}$ flux received on Earth can be determined following the procedures proposed in Refs. $[11,17,33]$. In this study it is assumed that the solar $\gamma^{*}$ originates from the Stueckelberg case with nondynamical mass, and its differential flux spectra in polarization functions are shown in Fig. 2 at certain $\left(m_{V}, \kappa\right)$. Experimental signatures of $\gamma^{*}$ are through their absorption and conversion to electrons in the detectors with a process analogous to the photoelectric effect. Consequently, the absorption rates of $\gamma^{*}$ inside a PPCGe detector are related to the photo-absorption cross sections in the germanium crystal. The energy is deposited locally due to the short range of ionized electrons inside PPCGe detectors. The expected energy spectrum of the solar $\gamma^{*}$ in a germanium detector is described by [17]

$$
\frac{d R(E)}{d E}=\frac{M}{\rho} \frac{E}{m_{V}}\left(\frac{d \Phi_{T}}{d E} \Gamma_{T}+\frac{d \Phi_{L}}{d E} \Gamma_{L}\right) B_{R},
$$

where $M$ and $\rho$ represent the mass and density of the germanium crystal, respectively, $E$ is the energy of $\gamma^{*}$, $\Gamma_{T, L}\left(m_{V}, \kappa\right)$ denote for the absorption rates for the $\gamma^{*}$ in transverse and longitudinal polarizations, $\left(d \Phi_{T, L} / d E\right) \times$ $\left(m_{V}, \kappa\right)$ are the differential fluxes on Earth with the different polarization functions mentioned above, while $B_{R}$ represents the branching ratio of the photoionization to

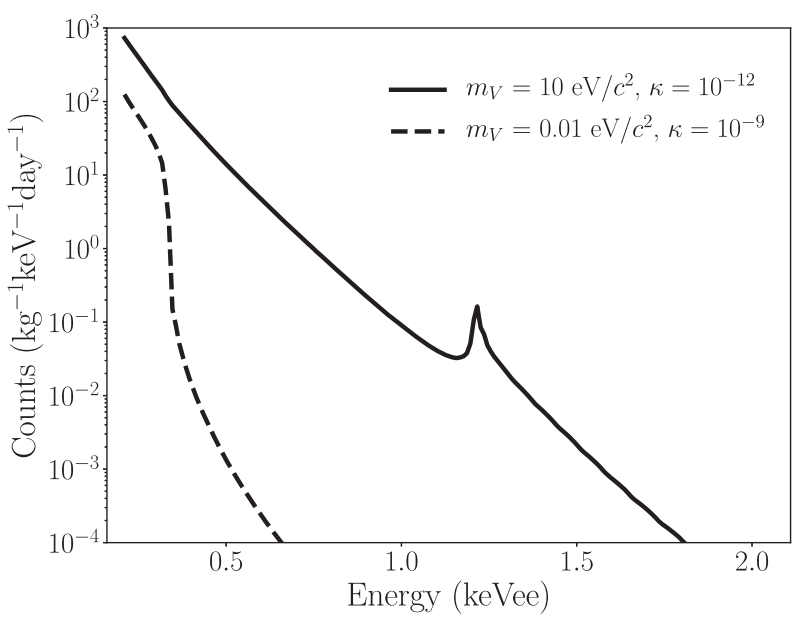

FIG. 3. The expected energy spectra of the solar $\gamma^{*}$ at the specified $\left(m_{V}, \kappa\right)$ in the CDEX-10 experiment.

the total absorption and it is equal to 1 for $m_{V}<300 \mathrm{eV} / c^{2}$ [17]. We neglect for simplicity the anisotropy of the germanium crystal in this analysis. The polarization functions for isotropic nonmagnetic materials can be derived from their optical refraction indices [34], based on which the absorption rates of transverse and longitudinal $\gamma^{*}$ are calculated according to the procedures described in Refs. [11,17,33].

Substituting the absorption rates and the differential fluxes of the solar $\gamma^{*}$ into Eq. (2), one gets the expected energy spectrum of the solar $\gamma^{*}$ in a germanium detector. Folding in the energy resolution of the detector, the expected energy spectra at two different $\left(m_{V}, \kappa\right)$ values are depicted in Fig. 3 as illustration.

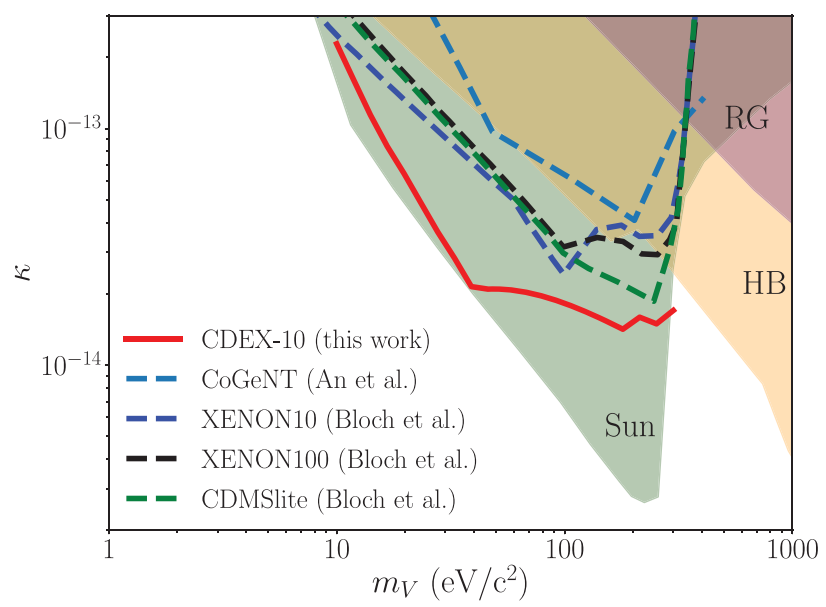

FIG. 4. Upper limits ( $90 \%$ C.L.) of $\kappa$ for solar $\gamma^{*}$ as a function of $m_{V}$. Results derived from direct detection experiments, i.e., CoGeNT [17], XENON, and CDMSLite [18], are shown as dashed lines. Shaded regions are the results from the astrophysical constraints including the Sun, horizontal branch stars (HB), and the red giant $(\mathrm{RG})[17,35,36]$. 


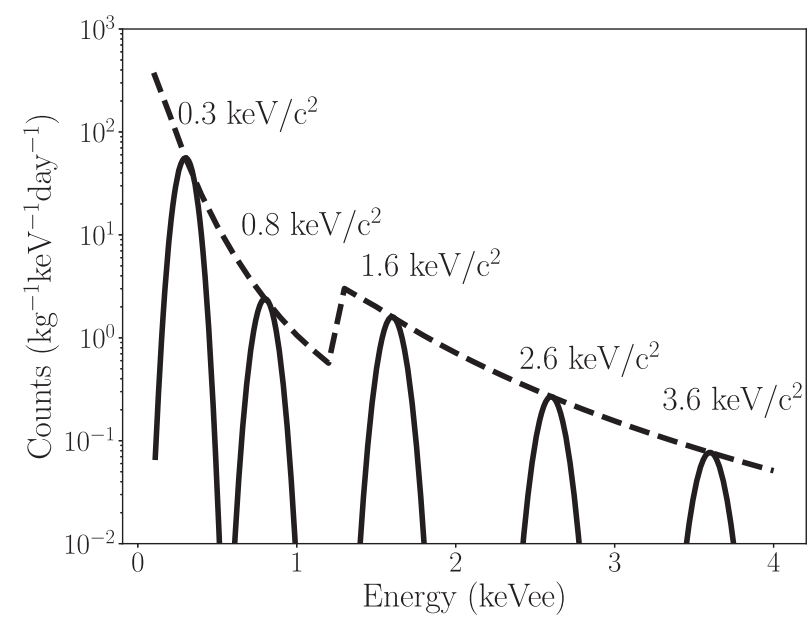

FIG. 5. The expected measurable energy spectra of $\gamma^{*} \mathrm{DM}$ at the $\mathrm{C} 10-\mathrm{B} 1$ detector with specific $m_{V}$ at $\kappa=10^{-14}$. The profile of energy peaks is depicted by a dashed line, similar to a photonelectric cross section curve.

No signals on solar $\gamma^{*}$ are observed with $\kappa$ at $1 \sigma$ excess from null effects for $m_{V} \sim 10-300 \mathrm{eV} / c^{2}$. Upper limits on $\kappa$ at $90 \%$ C.L. are displayed in Fig. 4, superimposed with previous results from direct detection experiments and astrophysical observations. The constraints from this work are the most stringent in $m_{V}$ from 10 to $300 \mathrm{eV} / c^{2}$, among the direct detection experiments to date.

The enhanced sensitivities of our measurement can be understood as follows. Experimental sensitivities are determined by the absorption rates $\Gamma_{T, L}\left(m_{V}, \kappa\right)$ of Eq. (2). At $E \sim O(10 \mathrm{eV})$ and $m_{V} \sim O\left(10 \mathrm{eV} / c^{2}\right)$, the absorption rates of germanium detectors is larger than xenon detectors for longitudinal solar $\gamma^{*}$, while the differences between the two nuclei are minor for transverse solar $\gamma^{*}$. In addition, the longitudinal $\gamma^{*}$ dominates the solar flux at $m_{V} \leq O\left(10 \mathrm{eV} / c^{2}\right)$, while the transverse component is larger at $m_{V} \geq O\left(10 \mathrm{eV} / c^{2}\right)[17,18]$. When $m_{V}$ increases, the main component of solar $\gamma^{*}$ evolves from longitudinal into transverse polarization. Consequently, C10-B1 with 160 eVee energy threshold has better sensitivity on both longitudinal and transverse $\gamma^{*}$, while the CoGeNT data [37] at $450 \mathrm{eV}$ threshold are not sensitive to the longitudinal component.

Dark photon dark matter.-The $\gamma^{*} \mathrm{DM}$ is also a possible contributor in experiments on rare event searches. Considering their number density and local velocity under DM halo assumption [38], the kinetic energy of $\gamma^{*} \mathrm{DM}$ is negligible compared with $m_{V}$ in the calculation of energy deposition. Using the same absorption rate of $\gamma^{*} \mathrm{DM}$ derived in Ref. [11], the expected differential event rates in a detector can be calculated with Eq. (3) [16], and the expected spectra at specific $m_{V}$ are shown in Fig. 5 as illustration. The differential rate observable at detectors is

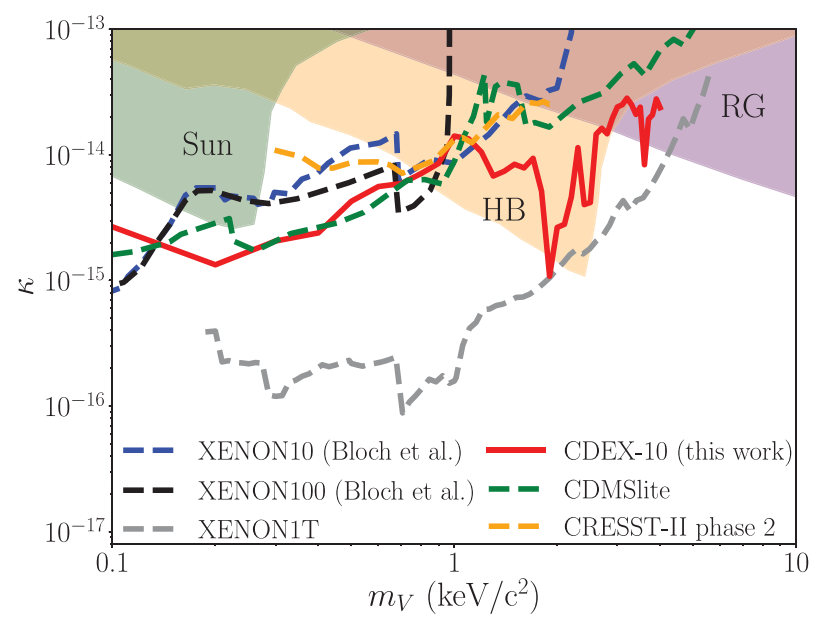

FIG. 6. Upper limits with $90 \%$ C.L. on $\kappa$ of $\gamma^{*}$ DM (solid red line) superimposed with the results from other direct DM searches (dash line). The constraints from CRESST-II phase-2 [14], XENON-10, XENON-100, CDMSlite [18], and XENON1T [19] are shown in orange, blue, black, green, and gray lines, respectively. The astrophysical constraints from anomalous energy loss in the Sun, horizontal branch stars, and red giant stars are also shown as shaded regions [16].

$$
\frac{d R}{d E}=V \rho_{\chi} \kappa^{2} I_{r},
$$

where $V$ is the volume of a detector, $\rho_{\chi}$ denotes for the local DM density (taken to be $0.3 \mathrm{GeV} / \mathrm{cm}^{3}$ in this Letter) and $I_{r}$ represents the imaginary part of the square of the refraction index for certain material tabulated in Ref. [34].

Following similar $\chi^{2}$ minimization analysis discussed above, no significant signal of $\gamma^{*} \mathrm{DM}$ is observed. The upper limits on $\kappa$ at $90 \%$ C.L. from 0.1 to $4.0 \mathrm{keV} / c^{2}$ are determined and depicted in Fig. 6, superimposed with those from previous direct detection experiments and astrophysical observations. In particular, the most stringent constraint is at $m_{V}=200 \mathrm{eV} / c^{2}$ where $\kappa=1.3 \times 10^{-15}$. The complexities of the exclusion curve above 1 keVee can be attributed to the $L$-shell $\mathrm{x}$ rays of cosmogenic radionuclides such as ${ }^{68} \mathrm{Ge}$ and ${ }^{68} \mathrm{Ga}$.

Conclusion.-In this Letter, we report the results on the searches of $\gamma^{*}$ based on the data from the CDEX-10 experiment, with exposures of $205.4 \mathrm{~kg}$-day from the C10-B1 detector and $244.2 \mathrm{~kg}$-day from the $\mathrm{C} 10-\mathrm{C} 1$ detector. A multidetector joint analysis is implemented for a $\gamma^{*} \mathrm{DM}$ search. No significant signal is found and the most stringent direct detection limits on $\kappa$ are set for solar $\gamma^{*}$ with $m_{V}$ of 10 to $300 \mathrm{eV} / c^{2}$. New parameter space of $\kappa$ for $\gamma^{*} \mathrm{DM}$ is probed within the $0.1-4.0 \mathrm{keV} / c^{2}$ mass region as well.

This work was supported by the National Key Research and Development Program of China (Grant No. 2017YFA0402200) and the National Natural Science Foundation of China (Grants No. 11725522, 
No. 11675088, and No. 11475099) and the Tsinghua University Initiative Scientific Research Program (Grant No. 20197050007).

Z.S. and L.P. J. contributed equally to this work.

*Corresponding author. yueq@mail.tsinghua.edu.cn Corresponding author. mahao@mail.tsinghua.edu.cn ${ }^{\ddagger}$ Corresponding author. yanglt@mail.tsinghua.edu.cn ${ }^{\S}$ Participating as a member of TEXONO Collaboration.

[1] B. L. Young, Front. Phys. 12, 121201 (2017).

[2] K. A. Olive et al. (Particle Data Group), Chin. Phys. C 38, 090001 (2014).

[3] C. Boehm and P. Fayet, Nucl. Phys. B683, 219 (2004).

[4] M. Pospelov, A. Ritz, and M. Voloshin, Phys. Rev. D 78, 115012 (2008).

[5] J. Jaeckel and A. Ringwald, Annu. Rev. Nucl. Part. Sci. 60, 405 (2010).

[6] J. Jaeckel, Frascati Phys. Ser. 56, 172 (2012).

[7] B. Holdom, Phys. Lett. B 166, 196 (1986).

[8] M. Ahlers, H. Gies, J. Jaeckel, J. Redondo, and A. Ringwald, Phys. Rev. D 77, 095001 (2008).

[9] M. Schwarz, E.-A. Knabbe, A. Lindner, J. Redondo, A. Ringwald, M. Schneide, J. Susol, and G. Wiedemann, J. Cosmol. Astropart. Phys. 08 (2015) 011.

[10] J. Redondo, J. Cosmol. Astropart. Phys. 07 (2008) 008.

[11] H. An, M. Pospelov, and J. Pradler, Phys. Lett. B 725, 190 (2013).

[12] A. Mirizzi, J. Redondo, and G. Sigl, J. Cosmol. Astropart. Phys. 03 (2009) 026.

[13] A. Aguilar-Arevalo et al. (DAMIC Collaboration), Phys. Rev. Lett. 123, 181802 (2019).

[14] G. Angloher et al., Eur. Phys. J. C 77, 299 (2017).

[15] S. N. Gninenko and J. Redondo, Phys. Lett. B 664, 180 (2008).

[16] H. An, M. Pospelov, J. Pradler, and A. Ritz, Phys. Lett. B 747, 331 (2015).
[17] H. An, M. Pospelov, and J. Pradler, Phys. Rev. Lett. 111, 041302 (2013).

[18] I. M. Bloch, R. Essig, K. Tobioka, T. Volansky, and T.-T. Yu, J. High Energy Phys. 06 (2017) 87.

[19] E. Aprile et al. (XENON Collaboration), Phys. Rev. Lett. 123, 251801 (2019).

[20] O. Abramoff et al. (SENSEI Collaboration), Phys. Rev. Lett. 122, 161801 (2019).

[21] K. J. Kang et al. (CDEX Collaboration), Front. Phys. 8, 412 (2013).

[22] J. P. Cheng et al., Annu. Rev. Nucl. Part. Sci. 67, 231 (2017).

[23] Y. C. Wu et al. (CDEX Collaboration), Chin. Phys. C 37, 086001 (2013).

[24] L. T. Yang et al. (CDEX Collaboration), Phys. Rev. Lett. 123, 221301 (2019).

[25] Z. Z. Liu et al. (CDEX Collaboration), Phys. Rev. Lett. 123, 161301 (2019).

[26] H. Jiang et al. (CDEX Collaboration), Phys. Rev. Lett. 120, 241301 (2018).

[27] H. Jiang et al. (CDEX Collaboration), Sci. China-Phys. Mech. Astron. 62, 31012 (2019).

[28] L. T. Yang et al., Nucl. Instrum. Methods Phys. Res., Sect A 886, 13 (2018).

[29] L. T. Yang et al. (CDEX Collaboration), Chin. Phys. C 42, 023002 (2018).

[30] Q. Yue et al. (CDEX Collaboration), Phys. Rev. D 90, 091701 (2014).

[31] J. N. Bahcall, Phys. Rev. 132, 362 (1963).

[32] G. J. Feldman and R. D. Cousins, Phys. Rev. D 57, 3873 (1998).

[33] R. Horvat, D. Kekez, M. Krčmar, Z. Krečak, and A. Ljubičić, Phys. Lett. B 721, 220 (2013).

[34] B. L. Henke, E. M. Gullikson, and J. C. Davis, At. Data Nucl. Data Tables 54, 181 (1993).

[35] P. Gondolo and G. G. Raffelt, Phys. Rev. D 79, 107301 (2009).

[36] J. Redondo and G. Raffelt, J. Cosmol. Astropart. Phys. 08 (2013) 034.

[37] C. E. Aalseth et al. (CoGeNT Collaboration), Phys. Rev. Lett. 106, 131301 (2011).

[38] J. F. Navarro, C. S. Frenk, and S. D. M. White, Astrophys. J. 490, 493 (1997). 